\title{
Enhanced Hydrate Nucleation Near the Limit of Stability
}

\author{
Felipe Jiménez-Ángeles ${ }^{\dagger}$ and Abbas Firoozabadi ${ }^{*, \dagger, \ddagger}$
}

Reservoir Engineering Research Institute, Palo Alto, CA 94301, USA, and Department of

Chemical and Environmental Engineering, Yale University, New Haven, CT 06510, USA

E-mail: abbas.firoozabadi@yale.edu

Phone: +1 (650)326-9172. Fax: +1 (650) 472-9285

Supporting Information

${ }^{*}$ To whom correspondence should be addressed

${ }^{\dagger}$ Reservoir Engineering Research Institute

${ }^{\ddagger}$ Yale University 
Table S1: Relevant data of various simulations. Nucleation times of propane aqueous solutions at different conditions. First and second columns are the label and number of runs, respectively, $N_{p}$ is the number of propane molecules, $N_{w}$ is the number of water molecules, $T$ is the temperature, $x_{p}$ is the propane mole fraction, $\tau_{n}$ is the nucleation time, and $j$ is the nucleation rate computed from $j=1 /\left(\tau_{n} V\right)$, where $V$ is the volume of the simulation box. The initial side length of the simulation box in $\mathrm{F}$ is $l=8.2 \mathrm{~nm}$ and $l=6.5 \mathrm{~nm}$ in all the other setups. $P=500$ bar.

\begin{tabular}{cccccccc}
\hline \hline Label & \# of runs & $N_{p}$ & $N_{w}$ & $T[\mathrm{~K}]$ & $x_{p}$ & $\tau_{n}[\mathrm{~ns}]$ & $j\left[\mathrm{~nm}^{-3} \mathrm{~ns}^{-1}\right]$ \\
\hline A & 2 & 326 & 6600 & 275.15 & 0.047 & $>500$ & - \\
B & 2 & 333 & 6600 & 275.15 & 0.048 & $>500$ & - \\
\hline C & 2 & 352 & 6600 & 273.15 & 0.050 & $>500$ & - \\
D & 3 & 342 & 6600 & 273.15 & 0.049 & $25,45,120$ & $7.3 \times 10^{-5}$ \\
E & 2 & 332 & 6600 & 273.15 & 0.048 & 48,95 & $6.4 \times 10^{-5}$ \\
F & 1 & 664 & 13200 & 273.15 & 0.048 & 120 & $3.8 \times 10^{-5}$ \\
G & 2 & 318 & 6600 & 273.15 & 0.045 & $>500$ & - \\
\hline H & 3 & 352 & 6600 & 270.15 & 0.050 & $3.5,4,17.4$ & $5.5 \times 10^{-4}$ \\
I & 2 & 342 & 6600 & 270.15 & 0.049 & 6,28 & $2.7 \times 10^{-4}$ \\
J & 2 & 312 & 6600 & 270.15 & 0.045 & 78,190 & $3.4 \times 10^{-5}$ \\
K & 2 & 288 & 6600 & 270.15 & 0.041 & 154,168 & $2.8 \times 10^{-5}$ \\
L & 2 & 270 & 6600 & 270.15 & 0.039 & $>500$ & - \\
\hline
\end{tabular}

Figure S1 shows the potential energy as a function of time for three independent simulations all for $x_{p}=0.048, T=273.15$ and $P=500$ bar. From these three simulations the average induction time is $\tau_{n}=87 \mathrm{~ns}$. 


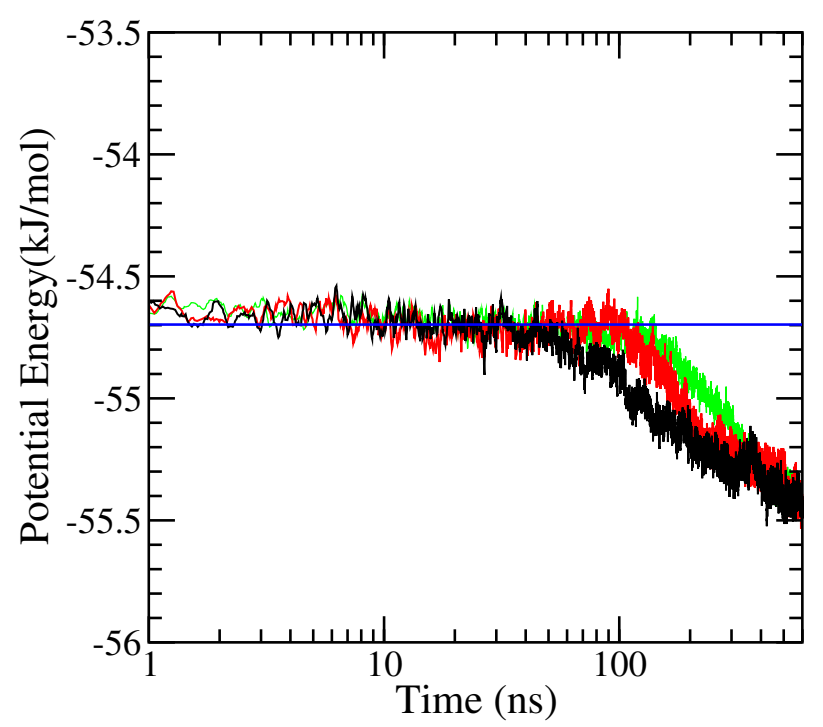

Figure S1:Potential energy vs. time for three systems with the propane concentration of $x_{p}=0.048$. In two simulations, $N_{w}=6600$ and $N_{p}=332$ (black and red lines); in the third simulation (green line) $N_{w}=13200$ and $N_{p}=664$. A horizontal straight line is drawn to guide the eye through the average constant values of energy. $T=273.15 \mathrm{~K}$, and $P=500$ bar.

Alternatively to $N_{5}$ (number of connected pentagonal faces), the size of a hydrate cluster can be defined by the number of hydrate-like water molecules forming the cluster $N_{C}$. A hydrate-like water molecule is part of the cluster if it is within a distance of $3.5 \AA$ of any water molecule belonging to the cluster. A water molecule can be part of only one cluster. Hydratelike water molecules are identified based on the value of their tetrahedral order parameter defined $^{1}$ as $q=\sum_{i=1}^{n_{i}}\left(\left|\cos \theta_{i}\right| \cos \theta_{i}+1 / 9\right)^{2}$, where $\theta_{i}$ is the angle formed by the bonds of oxygen atom of the test water molecule with two of its nearest neighbors and $n_{i}$ is the number triplets. By definition $q \geq 0$; a water molecule is closer to a tetrahedral configuration as its tetrahedral order parameter is closer to zero. Figure S2 shows the instantaneous size of the largest clusters during propane hydrate nucleation calculated using two different criteria of the tetrahedral order parameter, namely, $q<0.1$ and $q<0.4$. It can be seen that the cluster size depends on the value of $q$. In nucleation of methane hydrates $q<0.4$ is the criterion used to classify hydrate-like water molecules. ${ }^{2}$ Here for propane hydrates we see that with $q<0.4$ the cluster includes many water molecules which are not in a hydrate structure. 


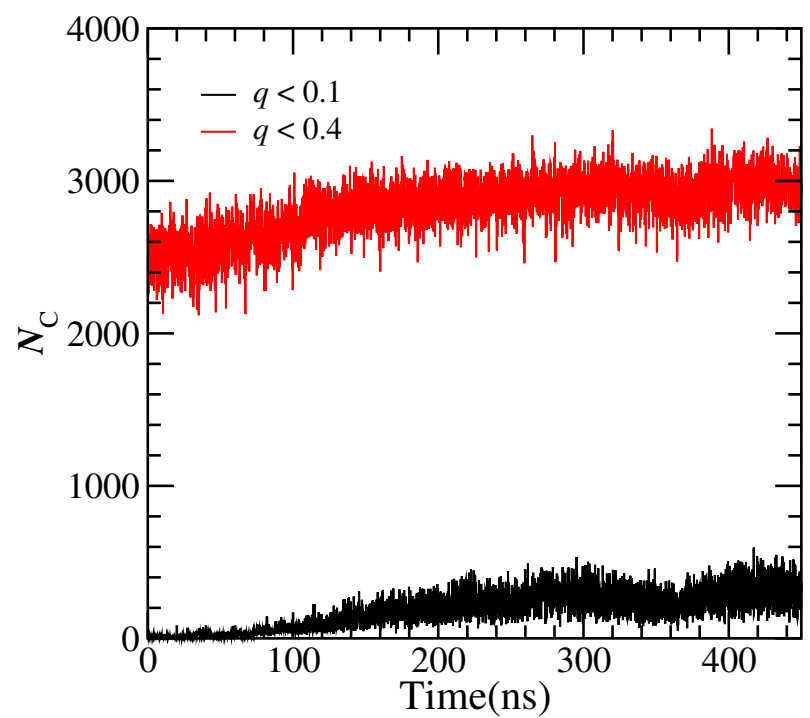

Figure S2: The instantaneous size of the largest clusters during hydrate nucleation calculated using the tetrahedral order parameter introduced by Báez and Clancy. ${ }^{1}$ The cluster size is computed using two different criteria of the tetrahedral order parameter, namely, $q<0.1$ and $q<0.4 . N_{w}=6600, N_{p}=332\left(x_{p}=0.048\right), T=273.15 \mathrm{~K}$, and $P=500$ bar.

For $q<0.1$, the cluster size as a function of time is qualitatively similar to the cluster size obtained with $N_{5}$ (see Figure 3 and discussion) which is consistent with a higher tetrahedral ordering of water molecules at lower values of $q$. Huge differences are observed: For $q<0.4$ half of the molecules are considered forming the hydrate cluster from the beginning of the simulation whereas for $q<0.1$ the size of the cluster consistently increases with the number of cages.

\section{References}

(1) Baez, L.; Clancy, P. Ann. N.Y. Acad. Sci. 1994, 715, 177-186.

(2) Sarupria, S.; Debenedetti, P. G. J. Phys. Chem. Lett. 2012, 3, 2942-2947. 